\title{
Introducing an Optimal (and a Simpler) Approach to Partial Least Squares Analyses
}

\author{
Dimitri Simonin and Bernard Morard
}

\begin{abstract}
The purpose of this study is to show the many possibilities that partial least squares (PLS) analysis offers, as well as its ease of use. This analysis is a useful tool, because it brings an additional point of view to statistical analysis beyond that of structural equation modeling analysis. Here, the authors suggest using a different approach to PLS, called "optimal PLS." It combines principal component analysis and PLS analysis to compute the data; by convergent iterations, this approach produces an optimal model not based on a reference model to best explain a given situation. The study illustrates this approach with two practical applications that create optimal models from the ground up: one in management controlling and the other in marketing. The software, which is used as a computational tool, has an algorithm based on optimal PLS. The study is original, because it chooses two opposing fields of research, namely management controlling (a quantitative discipline) and consumer behavior research (a qualitative discipline), in an attempt to understand when optimal PLS provides reliable results. The authors conclude that the use of PLS is multifaceted, and optimal PLS has a high capacity to explain the actual components, which helps researchers and analysts reach appropriate strategic decisions. With regard to the study's practical implications, the overview and the accompanying explanations will enable academics and analysts to use PLS analysis more easily by means of optimal PLS approach's five steps. They can add PLS and optimal PLS to their list of analytical tools to bring fresh points of view to their research.
\end{abstract}

Index Terms-Partial least squares path modeling, optimal PLS, optimal strategy, marketing research, consumer behavior, management controlling, algorithm, software.

\section{INTRODUCTION}

The main purpose of this article is to demonstrate the polyvalence and simplicity of a PLS analysis. PLS, also called "partial least squares for structural equation modeling" (PLS-SEM), is a quite recent statistical tool, of which partial least squares path modeling (PLS-PM) is a sub-part of structural equation modeling (SEM). For simplicity's sake, the term PLS will be used in this study as a generic label to cover all types of PLS analysis, including PLS-SEM and

Manuscript received December 17, 2016; revised February 5, 2017 This work was supported in part by the Faculty of Economics \& Management and the HEC Executive, University of Geneva, Geneva, Switzerland

D. Simonin is with the Marketing Discipline Group, University of Technology Sydney Business School, NSW, Australia (e-mail: dimitri.simonin@outlook.com).

B. Morard is with the Faculty of Economics \& Management, University of Geneva, Switzerland (e-mail: bernard.morard@unige.ch).
PLS-PM. At the start, PLS was mainly used in disciplines that analyze the principle of cause and effect, such as chemistry and physics. As PLS is particularly complex in a statistical context, most of the researchers presently using standard statistics like SEM are not interested in this tool with regard to computing data analysis and interpreting the results. Recently, a derivation of PLS, called optimal PLS (Jeannette, 2014; Morard et al., 2015) [1], [2], was developed. Unlike the existing types of PLS analyses, it selects the best graph of all possible graphs that can be generated for given data. It is a set of covariances and regressions that converges to a model by using iterations. Principal component analysis (PCA) generates axes, and optimal PLS (O-PLS) selects those with the highest potential for explanation by summing up the data variance. The authors develop this study to highlight a simplified approach to computing a PLS analysis and the interesting capabilities of going farther by using O-PLS as an optimal finder tool. The authors illustrate their approach in two very different fields of research: management controlling and consumer behavior research.

The authors aim to show why the PLS method is "a silver bullet" (Hair et al., 2012) [3] when used correctly in certain situations; they also seek to prove that PLS, if correctly understood, must not be considered a "voodoo statistics" method (Sosok et al., 2009) [4], as researchers sometimes think of it. With simple explanations, well-defined steps, and concrete examples, the authors introduce O-PLS. The PLS Assistant software (Morard et al, 2007) [5] and the website optimal-pls.com [6] will be used in the data analysis of O-PLS processes to ensure a simplified and understandable analytical process.

In summary, the broad outlines of this study are the following: First, the authors introduce the PLS analysis via a brief history and specificities that they compare with the SEM analysis. Second, they explain the different characteristics from a technical point of view. The PLS algorithm is briefly explained, and the O-PLS algorithm highlighted. Third, the authors enumerate and describe some of the main quality criteria that should be used to test the validity of the results of an SEM analysis and of every PLS analysis, including O-PLS, PLS-SEM, and PLS-PM. Fourth, they explain the five steps that need to be followed in order to use O-PLS as a simpler method than general PLS analyses. This method shows that researchers who do not have deep statistical competences can easily gain a statistical understanding of PLS methods. The authors suggest applying O-PLS analyses to management controlling and consumer behavior research, and using PLS Assistant to do this. Fifth, the authors provide some criticism and mention some of the limitations of the PLS 
analysis, and, more specifically, of O-PLS. They conclude with suggestions for further research to simplify the PLS analyses. The authors believe that if a broad range of academics were to have a better understanding the PLS analyses tools, it would be used in a wider range of business research fields. It would consequently produce interesting outcomes closer to the market reality and that react faster to changes in the markets.

\section{HISTORY}

In 1966, Herman Wold [7] published an academic paper on principal component analysis (PCA). He introduced the idea of PLS with the "nonlinear estimation by iterative least squares" (NILES) algorithm. In 1973, Wold renamed the algorithm NIPALS, the "nonlinear iterative partial least squares" algorithm. The main purpose of his research was to estimate structural equation models on latent variables (Tenenhaus, 1998) [8]. At the time, it was a general representation of the PLS regression.

Between 1983 and 1985, Harald Martens, along with Herman and Svante Wold, published the finalized algorithm called "partial least squares." This algorithm could solve the problem of having too many independent variables when using linear regression (Tenenhaus, 1998; Tenenhaus et al., 2005) [8], [9]. Herman Wold described SEM as 'hard modeling' with heavy distribution assumptions requiring several hundreds of cases. Conversely, he described and PLS as 'soft modeling' with requiring very few distribution assumptions, meaning that just a few cases were sufficient (Tenenhause et al., 2005) [9]. In 1990, Stone and Brooks presented the idea of using PLS in a "continuum regression" environment, which gave it a major boost as a statistical tool (Vancolen, 2004) [10].

In 1987, Lohmöller released the first software, LVPLS 1.8 , based on the PLS regression. Since then, researchers and authors have developed a couple of derivations of PLS software, although they needed particular software skills, depending on their own research field. Eventually, only a few software programs were developed, among which PLSGraph and Smart-PLS are the best-known, have led to the most significant advances in PLS software, and are used by a larger number of academic researchers. Since 2007, Morard, Jeannette, and Stancu [5] have worked on the development of a software called PLS Assistant (PLS-A), which uses the optimal PLS algorithm with the PLS algorithm as a foundation. Because it combines the statistical methods PLS and PCA into a single tool, PLS-A provides added value compared with other kinds of software on the market. It allows users to "compute the principal component analysis (PCA), estimate the path weighting scheme and, finally, generate bootstrap validation procedure and assess the best model from all possible graphs" (Morard et al. 2015, p. 304) [2]. In other words, it computes the number of axes, the latent variables needed to build a new model, and the connections between the axes, as well as allowing the interpretation of the axes. As PLS-A goes farther than PLS analysis by generating an optimal graph to explain the analyzed data, the term "optimal partial least squares" (O-PLS) is used in this study to refer to the algorithm related to the software.

\section{GENERAL OVERVIEW}

PLS is a multiple regression analysis and "a family of alternating least squares algorithms, or 'prescriptions,' which extend principal component and canonical correlation analysis" (Henseler et al., 2009, p. 284) [11]. It is perfectly adapted to predictive causal analysis in a complex situation that has only little theoretical information (Fernandes, 2012) [12]. Furthermore, the analyzing situation should manifest variables highly correlated with each other and in a large number (Vancolen, 2004) [10]. For instance, many managerial and industrial issues comprise "in-variables" and "out-variables." In these cases, the best would be to build relationships between these two types of variables without referring to any existent theoretical model. Moreover, most of the time, the "in-variables" are far more than the observations. With this kind of problem, common linear regression methods cannot solve the problem. Therefore, PLS regression is used (Tenenhaus, 1998) [8].

As Morard et al. (2015, p. 303) [2] argue, PLS has "its inherent limitations [among which the most notable is] that it is a limited information technique, designed to maximize prediction, rather than fit." But it shows a potential management use as a statistical analysis, because PLS analysis can make a prediction without a large data sample (Morard and Stancu, 2005) [13]. Currently, PLS is beginning to be used in all academic fields where it is difficult to collect sufficiently large data samples, such as marketing, strategic management, management controlling, and even accounting.

Compared with PLS analysis, structural equation modeling (SEM) is quantitative research analysis (DeVault, 2007) [14]. SEM analysis is much more popular than PLS analysis. Most of the time, researchers use SEM to confirm a hypothesis and not to explain an analyzed situation. SEM confirms hypotheses by means of a measurable data derivation (Lei and $\mathrm{Wu}, 2007)$ [15]. Nevertheless, PLS has the ability to research a model. The goal of using SEM is to distinguish between unmeasurable data and measurable data by using complex computation. From a technical point of view, SEM builds an estimation of unknown coefficients by means of linear structure equations computations. Two types of variables are considered in the model: observed variables and unmeasured latent variables. They correlate with each other (MacLean and Gray, 1998) [16]. SEM anchors its computation in a hypothesis, namely that causality links the unmeasured latent variables.

Helped by computers and adapted software, the use of SEM has become commonplace in most academic fields, including marketing research. However, DeVault (2007) [14] reminds SEM users of the "sample size rule of thumb." In order to ensure that the model is reliable enough, SEM requires ten to 20 times more observations than variables. Normally, 200 observations are required to have valid computations and prevent inaccurate conclusions (Medsker et al., 1994) [17]. Conversely, PLS requires a more flexible sample size and allows a smaller N value. DeVault (2007) [14] explains that SEM cannot prove the "directionality" of the variables' relationships with the causality direction. Hox and Bechger (1994) [18] rightly point out that causality already exists in the correlation of the analyzed data before the analysis is performed. This means that SEM cannot 
create the causality, nor does it test the existence of causality, because causality is implicit. Finally, SEM will always be an approximated reality, as it uses mathematical linear relations in the computation (DeVault, 2007) [14]. Working on the development of models in accounting and controlling, Morard et al. (2015) [2] add another layer by arguing that any researcher could make collected data fit any generated model if this is "necessary" to complete the study. In other words, depending on the chosen model, the estimation will always be considered correct. "A model will always only be a representation of the reality: a generalization of a multiplicity of realities" (Morard and Simonin, p. 413) [19].

In conclusion, "PLS is a soft modeling approach to SEM with no assumptions about data distribution" (Wong, 2013, p. 3) [20] and can be applied when there is a small sample size, when there is little available theory, predictive accuracy is crucial, and the specificities of the model have not been proved (Wong, 2013) [20]. When SEM is not applicable for the reasons cited above, PLS is a convenient tool that provides reliable conclusions (Hwang et al., 2010) [21].

\section{PLS CHARACTERISTICS AND ALGORITHM}

PLS is mainly used to explore and find a new model, because "PLS is claimed to explain at best the residual variance of the latent variables and, potentially, also of the manifest variables in any regression run in the model" (Esposito Vinzi et al., 2010, p. 48) [22].

PLS is composed of two cooperating models: an internal (structural) model, known as the "inner model," and an external (measurement) or "outer" model. The PLS algorithm is a succession of correlations and regressions by means of iterations. O-PLS goes farther by searching for the optimum model by means of a convergence of the data results. The number of iterations varies and depends on the analyzed data's complexity.

The schema below conceptualizes the basic PLS concept and illustrate an example of PLS path modeling:

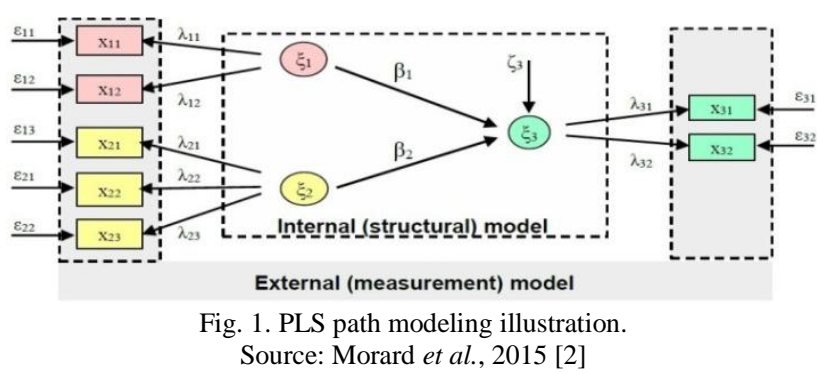

The internal model defines the causality between the latent (unobserved) variables (LVs). A linear equation resumes the causality:

$$
\zeta_{j}=\beta_{j 0}+\sum_{i} \beta_{j i} \zeta_{i}+v_{j}
$$

Source: Jeannette (2014, p. 50) [1]

The LVs are exogenous if related to other variables and endogenous if implying a regression to other variables. The beta $(\beta)$ coefficient represents the path coefficient, which correlates exogenous variables with endogenous variables. Zeta $(\zeta)$ is the residual vector, which is uncorrelated to any other vector. The particularity of this model is that there is no loop (Morard et al., 2015) [2].

The external model covers simple regression's connection between the measured (observed) variables (MVs) and their respective LVs. The former is composed of two types of models: the reflective measurement model and the formative measurement model (Henseler et al., 2009) [11] The following equation represents this connection:

$$
\begin{gathered}
x_{j h}=\pi_{j h 0}+\pi_{j h} \zeta_{i}+v_{j} \\
\text { Source: Jeannette (2014, p. 50) [1] }
\end{gathered}
$$

Furthermore, although LVs are a theoretical construct and cannot be observed, they can be formalized by a simple regression. "The assumption behind this model is that the residues, $\left[v_{J}\right]$, have a zero mean and [are] uncorrelated with the latent variable of the same block" (Morard et al., 2015, p. 303) [2].

The PLS model has three different types of links to the LVs. First, there are the links between all the LVs that comprise the inner model. Second, each of the MVs is related to an LV that comprises the outer model. Third, the weight relations of LVs are used to approximate the case value of a model's LV. This leads to a loop of four stages that make up the O-PLS algorithm (Jeannette, 2014) [1]:

1. External evaluation of the LV scores

2. Internal estimation of the weight of each LV

3. Internal evaluation of the LV scores

4. External estimation of the weight of each LV

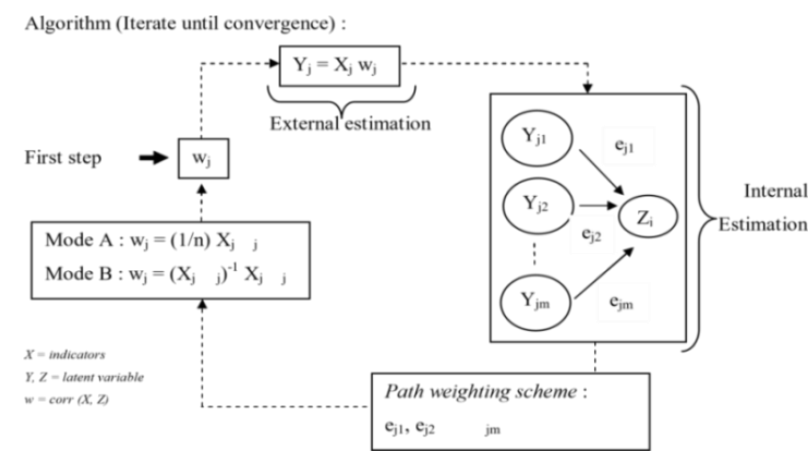

Fig. 2. O-PLS Algorithm schema Source: Morard (2014) [23]

On the schema, the choice of weight "e" is centroid, factorial or structural.

According to Jeannette (2014) [1], by using O-PLS, these stages have to iterate until they reach convergence, when:

$$
\sum_{h, j}\left|W_{j h}^{(k)}-W_{j h}^{(k-1)}\right|<10^{-5}
$$

Source: Jeannette (2014, p. 54) [1]

\section{QUALITY CRITERIA}

Several quality criteria are used to test the statistical reliability of the results. As PLS is a sub-part of SEM, some of the quality criteria are identical. Testing the model is crucial for the next steps of a PLS analysis to ensure that the 
resulting model is reliable enough to conduct a strategy analysis and to make decisions based on the model. Some of the main quality criteria are developed below.

\section{A. Cronbach's Alpha Coefficient}

Cronbach's alpha coefficient (CA) is commonly used to determine a model's reliability. It shows the level of homogeneity and uni-dimensionality. As it is a coefficient, the CA is between 0 and 1 . A model is considered to be homogeneous if the CA coefficient is larger than 0.7 (Esposito Vinzi et al., 2010) [22]. However, it is not suitable for a multidimensional structure, such as PLS, but rather for a SEM analysis situation. It becomes weak when it is used as a PLS quality criterion (Jeannette, 2014) [1].

$$
\alpha=\frac{N+\bar{r}}{(1+n-1) * \bar{r}}
$$

Source: Jeannette (2014, p. 55) [1]

where $N$ is the number of manifest variables and $\bar{r}$ the average correlation.

\section{B. Composite Reliability}

The composite reliability (CR) level, also called DillonGoldstein's rho, indicates the reliability of a summated scale and tests the external model's stability. The CR value is between 0 and 1 . The external model is statistically valid if the CR value is > 0.6 (Werts et al., 1974) [24]. The equation is:

$$
\rho=\frac{\left(\sum_{i=1}^{k} \lambda_{i j}\right)^{2} * \varphi_{j j}}{\left(\sum_{i=1}^{k_{j}} \lambda_{i j}\right)^{2} * \varphi_{j j}+\sum_{i=1}^{k_{j}} \theta_{i i}}
$$$$
\text { Source: Jeannette (2014, p. 56) [1] }
$$

with $\lambda_{i}$, the loading of a specific indicator $\mathrm{i} ; \varphi$, the empirical variance of the latent variable $\xi_{j}$; and $\theta_{\mathrm{ii}}$, the error variance of the $\mathrm{i}^{\mathrm{th}}$ indicator (Jeannette, 2014) [1].

It should be mentioned that the CR level is preferable to the CA coefficient. It does not assume that each manifest variable is equally weighted for each LV (Esposito Vinzi et al., 2010) [22]. "Consequently, the Cronbach's alpha is less efficient to measure reliability, whereas $\rho$ is a [closer] estimation" (Jeannette, 2014, p. 56) [1].

\section{Average Variance Extracted}

The average variance extracted (AVE) level represents the variance of the MVs explained by the common factor and tests the external model's stability. Developed by Fornell and Larcker (1981) [25], the AVE level considers that an LV has the ability to explain its indicator variance best. The value lies between 0 and 1. Chin (1998) [26] considers the external model to be statistically valid if the AVE value is > 0.5 ; in other words, if more than half of the variances are explained.

The AVE's equation is:

$$
A V E_{j}=\frac{\phi_{j j} \sum_{i=1}^{k_{j}} \lambda_{i j}^{2}}{\phi_{j j} \sum_{i=1}^{k_{j}} \lambda_{i j}^{2}+\sum_{i=1}^{k_{j}} \theta_{i i}}
$$

$k_{j}=$ number of indicators

$\lambda_{i j}=$ loading of the $\mathrm{i}^{\text {th }}$ indicator

$\phi_{j j}=$ empirical variance of the latent variable $\xi_{\mathrm{j}}$

$$
\begin{aligned}
& \theta_{i i}=\text { error variance of the } \mathrm{i}^{\text {th }} \text { indicator } \\
& \text { Source: Jeannette (2014, p. 57) [1] }
\end{aligned}
$$

\section{R-Squared}

The R-squared level tests the statistical stability of the internal model - in other words, the strength of the internal model. It is interesting to start with an R-squared test for each dependent LV when evaluating a PLS model's validity, "because the case values of the LVs are determined by the weight relations" (Chin, 1998, p. 316) [26]. If the value is > 0.67 , the model is strong; if the value is $>0.33$, the model is moderate; and if the value is $>0.19$, the model is weak (Chin, 1998) [26]. In a second step, the change in R-squared values can provide information about a specific independent LV's impact strength on a dependent LV (Chin, 1998) [26].

\section{E. Stability Test}

Specifically adapted for O-PLS analysis validation, the stability test, called bootstrapping, "is the technique of gauging components of an estimator (for example its variance) by measuring those aspects when sampling from an estimating distribution" (Morard et al., 2012, p.25) [27] and can be applied to all possible graphs (Bastien et al., 2005) [28]. This test is a nonparametric technique that estimates the shape, spread, distortion, and bias of a sample distribution in a particular statistical analysis (Jeannette, 2014) [1]. The bootstrap test is used with O-PLS to validate the procedure and to pick the best model from all the possible models. This model will have the best optimal cause-and-effect chain, meaning the highest forecast capabilities regarding the analyzed data and the most efficient strategy to follow (Morard et al., 2013) [29]. By testing each possibility to connect and arrange the different LVs, PLS-A applies bootstrapping to select the most stable generated PLS model (Morard et al., 2012) [27]. Yet, while the bootstrap quality criterion is statistically strong, it still has analytical limits, such as proving the connections between the indicators and ensuring a good AVE-explained score. In this respect, the CA and CR scores are statistically more accurate (Morard et al., 2013) [29].

It should be noted that "because the statistical [quality] tests [above] are based on the F-distribution, they are somewhat less sensitive to sample size" (Fornell and Larcker, 1981, p.47) [25]. This is one of the positive aspects of using PLS analysis instead of SEM.

\section{MODEl CONSTRUCTION}

According to Morard et al. (2012) [27], there are five chronological steps to take in order to build an optimal model and obtain optimal results using O-PLS. Variables are normalized after each step, and there are iterations until convergence emerges. Morard et al. (2015) [2] apply the results to determine the actual company strategy. Subsequently, the company strategy will be discussed and optimized if necessary. In data computation, there is always a presumption that an implicit model exists (Morard and Simonin, 2016) [19].

Step 1 - Collecting data: The researcher identifies and collects all the historical data and numerical elements that 
comprise the chosen quantitative indicators (financial, marketing, strategical, etc.) on which the analysis is built. The time slot should be as long as possible and homogeneous for all the variables (Morard et al., 2013) [29]. The whole analysis relies on this first step, because the collected data have to be reliable in order to obtain strong results. Consequently, this important step could take longer than the following steps (Morard et al., 2012) [27].

Step 2 - Cleaning data: The researcher cleans the collected data to avoid data errors that can distort the findings and lead to wrong conclusions. The data are selected according to the following criteria: "Reliability and consistency, same occurrence in time, ability to capture [the actual situation], information singularity, and, [finally,] clarity and straightforwardness" (Morard et al., 2012, p. 27) [27]. Only indispensable variables are retained in the further analysis (Morard et al., 2015) [2]. Moreover, the corrupted indicators are corrected and omitted from the analysis if not sufficiently corrected (Morard et al., 2013) [29].

This is an example of database final preparation and cleaning:

TABLE I: DATA PREPARATION AND CLEANING

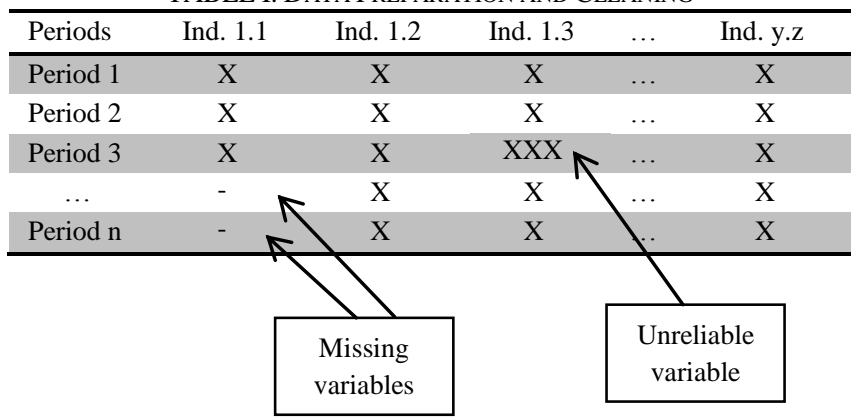

Source: Morard et al., 2015 [2]

Step 3 - Filtering and congregating variables: A number of axes are generated. By filtering, certain axes are selected, depending on whether there is a high correlation. Only the axes that contribute useful information and high correlation are kept. Subsequently, the information that the axes provide is brought together in indicators. Factor analysis and PCA are used to classify the indicators. The number of axes must provide at least $90 \%$ of the available information. Since each axis represents one data package, there should be an optimal layout between each data package (Morard et al., 2013) [29].

Filtering the indicators per axis:

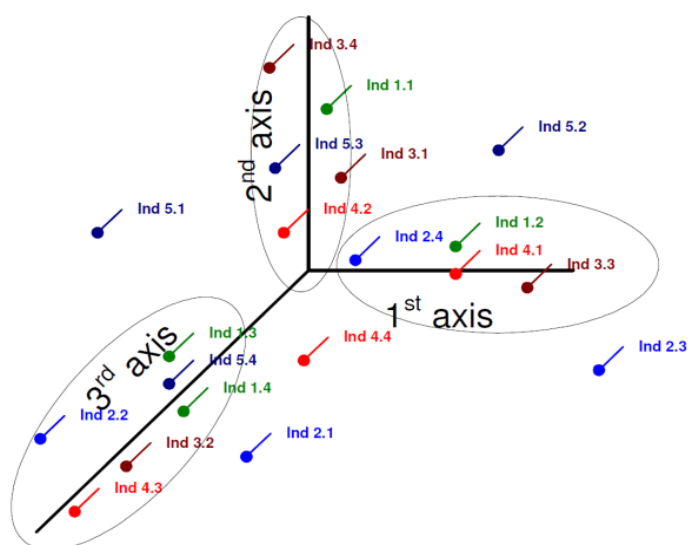

Fig. 3. Filtering the indicators per axis. Source: Morard et al., 2015 [2]
There are a few principles to follow when using PCA. First, the data are normalized, and the number of dimensions on the data chart should be reduced as far as possible. Second, the maximum amount of reliable information should be kept for the analysis. Third, interpretation of the axes should be easy and logical (Morard et al., 2013) [29].

Step 4 - Generating an optimal cause-and-effect schema: O-PLS regression is used to build a visual representation of the optimized situation with the cause-and-effect links between the variables, their directions, and the different indicators that make up each axis. The latter are used to name the axes, and the connections between axes are studied to understand and optimize the "cause-and-effect chain between the perspectives" (Morard et al., 2012, p. 30) [27]. Unlike other PLS models, O-PLS can determine the structure of the most viable and optimal model for the present situation.

In this step, the model should be tested for statistical reliability in order to determine the most statistically stable model. Statistical validation allows for analyzing the strength of all the internal and external models that PLS generates. This is possible because PLS is composed of structural equations from the outer model and from the inner model and because the relations between the data packages are quantified (Morard et al., 2013) [29]. For instance, the $\mathrm{R}$-squared level tests the statistical stability of the internal model (Chin, 1998) [26]. The AVE level, which indicates the variance in the MVs explained by the common factor, and the CR level, which indicates the reliability of a summated scale, test the stability of the external model (Chin, 1998; Werts et al., 1974) [26] - [24]. From a statistical point of view, the internal and external models can be strongly, moderately or weakly stable. The best way to confirm a model's statistical reliability is to use a variety of quality tests. By considering several tests instead of just one, each test confirms the quality result of the others (Morard and Simonin, 2016) [19].

Step 5 - Applying PLS equation: The structural equations from both the outer and the inner models that make up OPLS are applied "to study and forecast the relation for the long term" (Morard et al., 2012, p. 33) [27]. In other words, these equations allow the researcher to analyze the measures' variance impact on the entire model, forecast the future changes of the present situation, determine the composition of the elements necessary to bring about optimal changes, and to determine how these changes will impact the future situation (Morard et al., 2013) [29].

Morard, Stancu, and Jeannette $(2012 ; 2013 ; 2015)$ [27] [29] - [2] use this method in the field of accounting, but the same steps could also be taken in any other academic fields, such as marketing research and business strategy (Morard and Simonin, 2016) [19]. Furthermore, due to of the type of connection between latent variables, certain rules must be followed in order to build a model using O-PLS (Jeannette, 2014) [1]: There must be (1) no causal loop, (2) at least one connection per latent variable, (3) at least one assigned indicator per latent variable, (4) indicators that cannot be related to more than one latent variable, and (5) no submodel.

With these five easy steps, the authors prove that the use 
of PLS can be simplified by using O-PLS. This way of using PLS contradicts many recent authors' idea that researchers "do not feel comfortable with PLS because of their unfamiliarity and/or lack of understanding, with some going as far as likening PLS to "voodoo statistics"” (Sosik et al., 2009, p. 6) [4].

\section{PRACTICAL APPLICATIONS}

PLS is used in many fields, and researchers are increasingly convinced of this statistical tool's added value: Wong (2013) [20] lists Bass et al. (2003) in behavioral sciences, Henseler et al. (2009) [11] in marketing, Sosik et al. (2009) [4] in organization, Chin et al. (2003) in management information system, and Hulland (1999) in business strategy. PLS is used in applied research, particularly when the amount of data (i.e. the number of participants) is small and the data distribution is skewed (Wong, 2013) [20]. The authors chose to pick two very different fields to show the similarities that are visible when applying PLS analysis, and, more specifically, O-PLS using PLS-A and the website optimal-pls.com [6]. In both situations (management controlling and consumer behavior research), the goal is to find an optimal strategy. The management controlling case is concerned with finding an optimal operational strategy for a specific company, while the consumer behavior case focuses on finding a marketing strategy for consumers of a product in a specific market. The authors apply in-house software, called PLS-A, which is based on the PLS algorithm and uses bootstrapping validation. The authors bring these two very different fields closer in order to highlight the scope of PLS and O-PLS analysis use in academic research.

\section{A. Management Controlling Analysis}

The authors chose Kaplan and Norton's work on a generalized BSC, published in 1996, as an example that contradicts the O-PLS analysis. Kaplan and Norton argue that a BSC reflects a company's strategy through predefined axes and indicators, which can be applied to all companies (1996) [30]. Logically, it should be possible to extract the strategy by analyzing any company's BSC. Yet, since Kaplan and Norton's BSC has a generic application, such an extraction is impossible. Therefore, since the strategy of each company is different, a tailor-made BSC must be defined for each company based on its own data. According to Morard et al. (2012) [27], if the BSC is specific to the company, as well as determines and shows the key factors for strategic decision success, Kaplan and Norton's generic BSC is not suitable and does not have the same predictive capabilities. Usually, when an analysis of a company strategy is done, little data are available, which mutate quickly and are difficult to obtain. Since 2005, Morard and his research partners suggested using PLS to study the impact of changes on the company strategy and on the company itself. PLS is very effective, has great prediction skills, and can be adapted quickly to data modifications (Jeannette, 2014) [1]. Morard et al. (2012; 2013; 2015) [2], [27]-[29] use O-PLS analysis to determine the optimal balanced scorecard (OBSC). The goal is "not to develop the best indicators, which sometimes could be driven [by] subjectivity and personal preference, but to actually highlight the importance of the performance indicators available" (Morard et al., 2015, p. 69) [2]. Visual representation is generated by PLS-A as a strategic map that represents the actual strategy of a specific company. This information is used to build the OBSC (Morard et al., 2012; 2013; 2015) [2], [27]-[29] that reflects the company's optimal strategy at present. The goal is to use this information to build an optimized strategy for the company to follow in the near future. Following this analytical method, the authors note that the OBSC is optimal, because it reflects the company's most efficient strategy. It means that if each company has its own specific BSC, it also has its own specific strategy.

Below, the authors follow the whole process of computing random company data (Morard, 2014) [23] using PLS-A within the context of management controlling.

The first step uses PCA to explain the variance of the components. The authors chose a model with three axes (LVs), because PCA computation shows that three axes explain more than $90 \%$ of the total variance $(93.33 \%)$. The number of axes should not be more than ten, as a larger number would not adequately regroup the available information.

TABLE II: DATA OUTPUT WITH PCA COMPUTATION

\begin{tabular}{|c|c|}
\hline Number of Axes (components) & \% cumulative variance \\
\hline 2 & $82.89 \%$ \\
\hline$\underline{\mathbf{3}}$ & $\underline{\mathbf{9 3 . 3 3 \%}}$ \\
\hline 4 & $97.10 \%$ \\
\hline 5 & $99.25 \%$ \\
\hline 6 & $99.91 \%$ \\
\hline
\end{tabular}

Source: Morard (2014) [23] data output using PLS-A computation

After choosing the number of axes, PCA computation shows that normalized variables (i.e. indicators) have an influence on the three selected axes. The table below shows the extracted indicators from the components' matrix:

TABLE III: COMPONENTS MATRIX OF EXTRACTED INDICATORS

\begin{tabular}{|c|c|c|c|}
\hline Indicators (variable) & Axis 1 & Axis 2 & Axis 3 \\
\hline Sales & 0.367 & $\underline{\mathbf{0 . 4 1 0}}$ & 0.173 \\
\hline Gross profit & $\underline{\mathbf{0 . 3 8 3}}$ & 0.335 & 0.136 \\
\hline Customer credit & 0.337 & $\underline{\mathbf{0 . 4 2 5}}$ & 0.079 \\
\hline Delivery time & 0.367 & $\underline{\mathbf{0 . 4 1 0}}$ & 0.173 \\
\hline Market share changes & 0.288 & 0.379 & $\underline{\mathbf{0 . 5 1 6}}$ \\
\hline Absenteeism & 0.302 & 0.121 & $\underline{\mathbf{0 . 7 0 8}}$ \\
\hline Training (days) & $\underline{\mathbf{0 . 3 9 9}}$ & 0.252 & 0.282 \\
\hline Staff turnover & 0.370 & $\underline{\mathbf{0 . 3 8 6}}$ & 0.263 \\
\hline
\end{tabular}

Source: Morard (2014) [23] data output using PLS-A computation

The highest correlation number for each variable is selected, because the higher the number is, the more the variable influences the axis (Morard et al., 2015) [2]. It 
should be noted that if a variable does not influence an axis enough, it should be removed to avoid invalid outputs. Using PLS analysis, the last PLS-A computation produces the strategic map shown below:

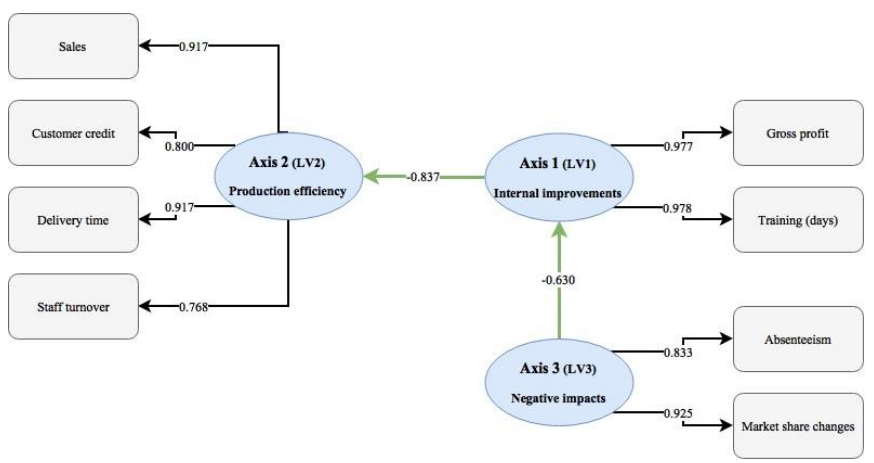

Fig. 4. Strategic map using PLS-A computation.

Source: Morard (2014) [23] data output using PLS-A computation

In the internal model, these axes are linked by greencolored arrows of correlations (correlation index). These are positive and negative and show the impact of one axis on another. In the external model, axes are also related to variables: the measurable variables (MVs). The black arrows indicate the correlation strength between the LVs and MVs. To name the three axes, the authors took into consideration which MVs are related to which axes; the axes' names reflect the theme of the related MVs. The strategic map can be understood as follows: If an indicator registers a change, the whole model will be impacted.

After the strategic map has been generated to represent the company's actual strategy, quality tests are used to validate the strength of the internal and external models generated by O-PLS. The table below shows the results of the quality tests:

TABLE IV: RESULTS OF QUALITY TESTS

\begin{tabular}{|c|c|c|c|c|}
\hline Axes & Name & CR & AVE & R-Squared \\
\hline Axis 1 & Internal improvement & 0.762 & 0.728 & - \\
\hline Axis 2 & Production efficiency & 0.873 & 0.775 & 0.698 \\
\hline Axis 3 & Negative impact & 0.978 & 0.956 & - \\
\hline
\end{tabular}

Source: Morard (2014) [23] data output using PLS-A computation

First, the composite reliability (CR) level, which is the reliability of a summated scale, tests the validity of the external model. In this OBSC, axis 1, axis 2, and axis 3 all have higher CR values than the 0.6 required by Chin (1998) [26] (0.762, 0.873, and 0.978, respectively). This means that, from a statistical point of view, the external model is statistically valid for the three axes. Second, the average variance extracted (AVE) level, which is the variance in the MVs explained by the common factor, tests the external model's stability. According to Wert et al. (1974) [24], the three axes in the OBSC have higher AVE values than required $(0.728,0.775$, and 0.956 , respectively). The external model is statistically strongly stable regarding the three axes. Third, the R-squared level of axis 1 (0.689), which tests the statistical stability of the internal model, determines that the optimal model for this OBSC is substantially explained from a statistical point of view (Chin, 1998) [26]. It should be noted that we only have an Rsquared value for axis 2, because only axis 1 and axis 3 have an impact on other axes. Thus, regarding the different values and results of the different quality criteria, the authors conclude that this generated model represents the best combination of a cause-and-effect relationship with regard to the bootstrap quality criterion (Morard et al., 2012) [27]. Thus, in terms of the data, this optimal model is the one with the best predictability capabilities for the actual company strategy.

As it is easy to generate a model, the advantage is that different scenarios of LVs' modifications can be tested and compared with each other to choose the best strategy in line with the company and the market. The model with the optimal path, the cause-and-effect chain, between axes (LVs) is computed by means of iterations. However, according to Jeannette (2014, p. 60) [1], "as [with] any statistical tool used, the prediction model should be considered more as trends rather than exactly future relevant values."

\section{B. Consumer Behavior Research}

Marketing researchers use PLS analytical tools for their unique evaluation strength. PLS can be applied at the beginning of a study, because it can test exploratory models with strong prediction skill. O-PLS is even able to build a model from data without a pre-existing model. Henseler $e t$ al. (2009) [11] add that the prediction-oriented function of PLS is a big advantage in marketing study, as it is used to build the theoretical part of the analysis when theory is lacking. Researchers thus save precious time when completing a valid analysis. Moreover, in many cases, the marketing researcher cannot collect enough data to complete the study. There are many reasons for this lack of data, including missing data, a lack of replies, and data survey accuracy. "PLS offers excellent capabilities for work with small samples and formative measurement, as the methodology is sufficient for most success factor (cause indicator) analyses in international marketing research" (Henseler et al., p. 311) [11]. In some cases, a distribution or model identification incompatibility could make the use of SEM impossible. Consequently, PLS is used as a substitute solution for a clean analysis (Henseler et al., 2009) [11].

From the point of view of Noyan and Simsek (2012) [31], who applied PLS analysis to determine a model of repurchase intention and consumer perception, there is a big advantage in using this "soft model" of analysis (PLS). Despite "the large sample, the non-normality of the variables, the large number of MVs and the complex nature of the proposed model" (Noyan and Simsek, 2012, p. 926) [31], the authors of this work succeeded in reaching accurate and reliable conclusions.

Morard and Simonin (2016) [19] used PLS, specifically O-PLS, in a context of consumer behavior analysis by building the optimal consumer behavior description (OCBD) computed by PLS-A; the authors built a tailor-made model of the e-consumer of wine without any pre-existing model. Their model was built from scratch and is aimed at precisely explaining consumer behavior in this specific market. "Being able to use statistics to generate an optimal, tailormade model helps to understand the reality of the field with 
greater precision" (Morard and Simonin, 2016, p. 416) [19]. For the sake of simplicity, the authors of the present paper skip the explanation of PLS-A's PCA computation process, because it is explained in the management controlling example. As shown in the output below, PLS allows the researcher to determine the axes depending on measurable, related variables (MVs):

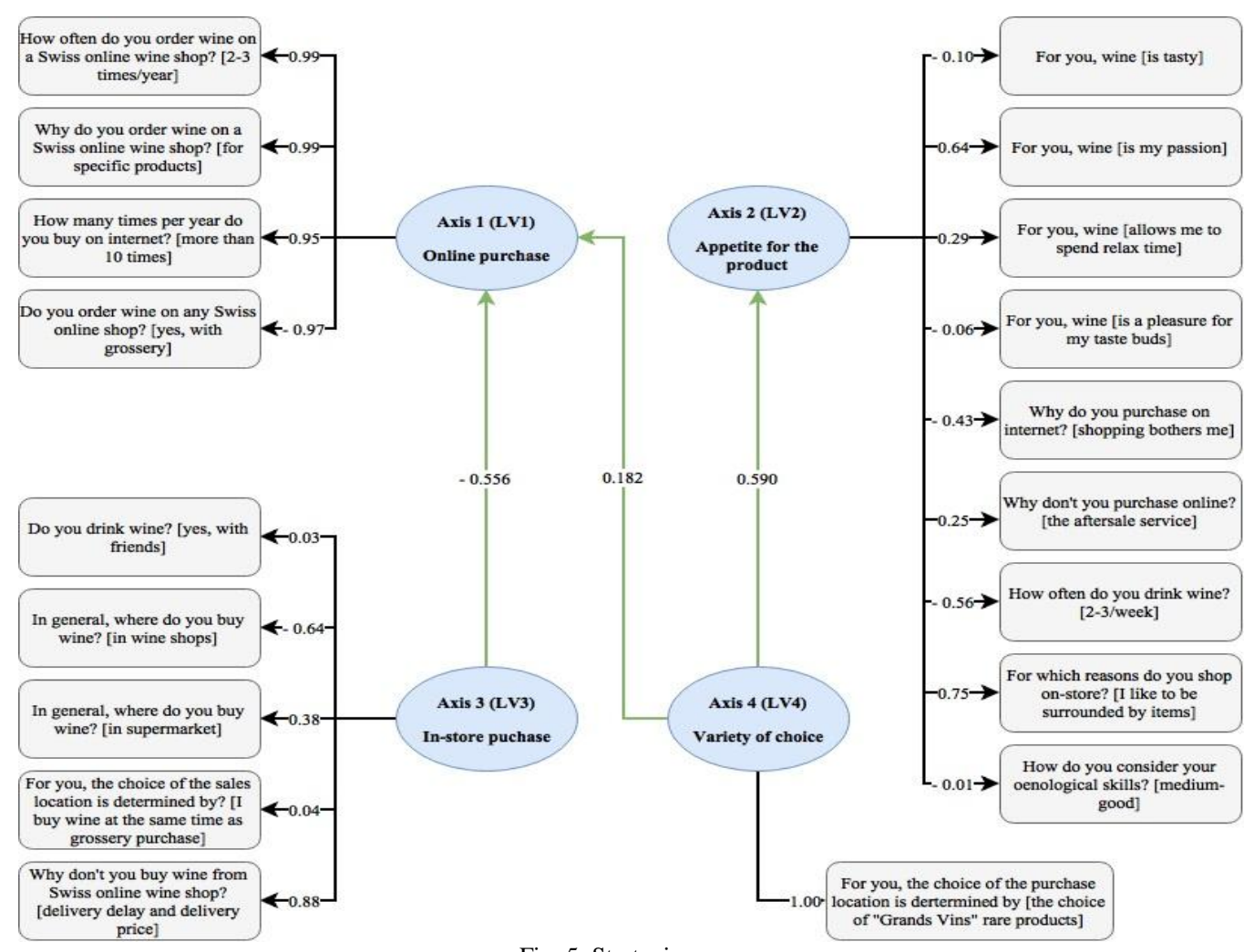

Fig. 5. Strategic map.

Source: Morard and Simonin (2016, p. 414) [19]

After taking into consideration which MVs are related to which axes, Morard and Simonin (2016, p. 415) [19] name axis 1 an online purchase, axis 2 the appetite for the product, axis 3 an in-store purchase, and axis 4 a variety of choice.

Thereafter, the researchers test the stability of their model using quality tests:

TABLE V: RESULTS OF QUALITY TESTS

\begin{tabular}{|c|c|c|c|c|}
\hline Axes & Name & CR & AVE & R-Squared \\
\hline Axis 1 & Online purchase & 0.825 & 0.940 & 0.369 \\
\hline Axis 2 & Appetite for the product & 0.085 & 0.185 & 0.348 \\
\hline Axis 3 & In-store purchase & 0.110 & 0.270 & - \\
\hline Axis 4 & Variety of choice & 1.000 & 1.000 & - \\
\hline
\end{tabular}

Source: Morard and Simonin (2016, p. 415) [19]

In this OCBD, the composite reliability (CR) level, which shows the reliability of a summated scale and tests the validity of the external model, and the average variance extracted (AVE) level, which indicates the variance in the MVs explained by the common factor and the stability of the external model, both have higher values than required for axis $1(0.825 ; 0.940)$ and axis $4(1.000 ; 1.000)$, according to Chin (1998) [26]. This means that the external model is statistically strong for axis 1 and axis 4 . Yet, the $\mathrm{AVE}$ value and the $\mathrm{CR}$ value are lower than required for axis $2(0.085 ; 0.185)$ and axis $3(0.110 ; 0.270)$, which means that the external model is statistically weak for axis 2 and axis 3 . With a very strong axis 1 and axis 4 and with a weak axis 2 and axis 3, Morard and Simonin (2016) [19] conclude that the external model is moderate from a statistical point of view. The R-squared values of axis 1 (0.369) and axis $2(0.348)$ show that the internal model is moderately stable from a statistical point of view (Werts et al., 1974) [24]. While the results do not fit perfectly with the required scales of the used quality test, the internal model is nonetheless statistically valid. Even if the model has moderate stability characteristics from a statistical point of view, Morard and Simonin (2016) [19] confirm that this optimal model is the model that is the best combination of cause-and-effect relationship with regard to the bootstrap quality criterion. This OCBD is the model that has the best predictability capabilities for the behavior of consumers making wine purchases online in Switzerland. After validity testing, Morard and Simonin (2016) [19] confirm that their model describes the behavior of a consumer in a specific market based on the cause-and-effect chains, namely the relations between the MVs and the LVs found in the analysis.

The authors think that an analogy can be made here comparing these two fields of research: Just as Morard et al. (2015) [2] make a deduction in a study in the field of management controlling, Morard and Simonin (2016) [19] argue that each market and each product has its specific strategy based on an optimal consumer behavior profile. Therefore, if each market has its own consumer profile, SEM and applications of traditional consumer behavior models cannot be applied, because they are generic and not optimal. A marketing strategy based on a consumer behavior analysis of a specific market is efficient and effective only if the consumer behavior profile is optimal. In 
other words, if a marketing strategy is built using O-PLS analysis, the data should only concern the consumer of the analyzed market, and the strategy should only be applied to this specific market.

In conclusion, the authors observe that O-PLS has a very large application scope in academic research. They infer that, if it can be used in research fields as divergent as management controlling and consumer behavior research, O-PLS can easily be applied to every research field where the conditions described by Wong (2013) [20] are respected. The PLS-A software is a tool that goes farther than PLS analysis does, and provides output in the form of a schemaa map that is by implication the optimal model based on the analyzed specific data. In various management fields, this map can be used to build a solid strategy, such as marketing strategy, business strategy, company strategy, and market strategy, as well as to forecast the impacts of changes.

SEM and PLS are obviously powerful tools that lead to interesting conclusions and applications. Yet, it also has several constraints. First, there are tools that need resources in terms of time, money, and work. In this case, conclusions are difficult and slow to obtain. The authors think that it is an important weakness, because research, economy, markets, and changes are moving fast. In some situations, during the time taken to make an analysis and conclusions and to mobilize the adequate financial resources, the reality has changed, and the analysis is no longer usable. Hence, an analysis must be made within a short time with conclusions that can be easily adapted. O-PLS shortens the analysis process by using the cause-and-effect links between the different variables. It takes very little time to run another analysis by modifying only a few data points. Thereafter, a new optimal model is used to adapt the strategy to the new reality. It also means that fewer financial resources are needed to reach reliable conclusions. For instance, in consumer analysis, a consumer behavior model and, thus, a company's entire marketing strategy can become obsolete in a few weeks if a competitor releases a successful product. Second, the authors are convinced that issues with a lack of data are common in analytical research. A large amount of reliable data is very hard to obtain, even with significant time and financial investments. PLS, as well as O-PLS, can be applied in a situation with little available data. For instance, in management controlling, useful and reliable data are difficult to select. The company size, too much disorganization in the available information, and restricted budgets are some of the issues encountered when providing a company analysis. Third, the authors highlight the fact that (unlike PLS) SEM is based on existing models. This means that the model based on the SEM analysis is generic. The model fits the analyses and the conclusions made, but the model that is applied is not optimal and, as DeVault (2007) [14] argues, "SEM will always be an approximated reality, as it uses mathematical linear relations in the computation" (Morard and Simonin, 2016, p. 413) [19]. OPLS analysis develops a model for each computed group of data.

\section{CRITICISM}

First, technically speaking, PLS, and more specifically O-
PLS, is not the holy grail of statistical analyses and cannot be applied in each case to substitute other statistical tools, such as SEM. The weaknesses of PLS, and of O-PLS, include the following: (a) In a case of a small sample size, the structural path coefficient must be big (Wong, 2013) [20]; (b) PLS does not resist multicollinearity, even if it reacts better to it than other methods do (Henseler et al., 2009) [11]. In fact, PLS generates internal and external models using multiple regression. This could contribute multicollinearity problems to the estimation; (c) There is no possibility of bi-directed correlations, as the arrows have only one direction (Wong, 2013) [20]; (d) PLS lacks a technical basis and can mainly be used in an "on-the-field" study. "Developing a more formal methodology [...] while using a simplified model" could be a feasible solution (Morard et al., 2013 p. 25) [29]; (e) Consistency latent variables' scores can lead to wrong conclusions regarding component estimation, loadings, and path coefficients (Wong, 2013) [20]; (f) PLS can "create large mean square errors in the estimation of path coefficient loading" (Wong, 2013 , p. 32) [20]. Second, because the O-PLS analysis translates and helps implement the strategic vision by means of a holistic approach, a focus on one of the analyzed elements is not viable without rethinking the whole analysis. Indeed, O-PLS uses the interdependency of the analyzed elements with cause-and-effect chains between these. Analyzing only one element would not be viable. Third, adaptability to and flexibility regarding the data and fields of analysis are a very positive aspect. Yet, the conclusions drawn from the results of an O-PLS analysis are not easily transferable, because they are tailored to the analyzed situation. An analysis must be carried out of each studied situation, which can be expensive.

Lastly, comparing a management controlling with a consumer behavior analysis, the authors noticed that PLS, and thus O-PLS, are less suitable for an analysis with coded qualitative data. It seems that the optimal model found by computing quantitative data is statistically more reliable and more stable than the optimal model based on qualitative data, which is moderately stable from a statistical point of view.

It is very important to take these issues into consideration, because they can lead to a study's failure. If the conclusions of the analysis are wrong, the model will not reflect the market reality. Practically speaking, if a company is not aligned with the market and implements a strategy or marketing strategy that cannot increase its competitiveness, it could face a difficult financial and economic situation. To avoid these issues, Henseler et al. (2009) [11] suggest using a large spectrum of statistical analysis methods in a single study. Each statistical method has different points of view, and the researcher can confirm or reject some of previous results and conclusions (Morard and Simonin, 2016) [19].

\section{CONCLUSION AND FURTHER RESEARCH}

The authors of this study did a simplified statistical overview of PLS and introduced the O-PLS approach. They explained five steps that help researchers understand clearly how to apply O-PLS in their research. Based on past studies 
and practical examples in two different research fields, management controlling (Morard, 2014) [23] and consumer behavior research (Morard and Simonin, 2016) [19], the authors aimed to show how easy and efficient O-PLS analysis is and how it can be used in various analysis situations. An O-PLS analysis of different types of research proves that PLS is not a "voodoo" statistics tool, as Sosik et al. (2009) [4] label it.

Finally, it is important to continue to explore the interesting possibilities that a PLS, analysis and, more specifically, O-PLS, offers business research. Generating an optimal model helps with a closer understanding of reality from a statistical point of view. Nevertheless, Morard et al. (2015, p. 307) [2] argue that the future challenge facing researchers using PLS is to reconcile "the pragmatism required by the organizations [with] the need for a more theoretical framework requested by researchers." Setting up the entire conclusion of an analysis on historical data and computing current data to suggest a "cause-and-effect" model could be considered idealistic. Analyzing the entire current situation is the only way to plan the future rationally (Morard et al., 2013) [29].

The authors believe it is important that future research should search for a way to simplify PLS methods to make it theoretically available for all researchers, because its complexity in application and in result understanding are the negative aspects of this method. In fact, most researchers have neither the ability nor the competence to understand how PLS works from a theoretical point of view and instinctively shy away from using the PLS approach. More research should therefore be done using PLS methods with qualitative data. The consumer behavior study using O-PLS is not conclusive and the authors question the statistical strength of O-PLS when subjectivity is part of the analysis. Finally, the authors suggest searching for new reliability tests to specifically use in a PLS analysis. If reliability tests are good, strong and tailored to PLS algorithms, researchers will be able to draw conclusions with greater confidence. In this context, the authors think that PLS can also appeal to those outside academia, like financial analysts, and be a fit for users with no theoretical knowledge of statistics. Given that O-PLS has the ability to synthesize the available information quickly, even when there is too much data to compute, it can be used in such contexts.

\section{REFERENCES}

[1] C. Jeannette, "Time evolution analysis of key performance indicators in an effective Balanced Scorecard," $\mathrm{PhD}$ thesis, University of Geneva, 2014.

[2] B. Morard, A. Stancu, and C. Jeannette, “A comparison between two balanced scorecards: optimal vs. Kaplan and Norton model," Journal of Economics, Business and Management, vol. 3, no. 2, pp. 302-308, 2015.

[3] J. Hair, M. Sarstedt, T. Pieper, and C. Ringle, "The use of partial least squares structural equation modeling in strategic management research: A review of past practices and recommendations for future applications," Long Range Planning, vol. 45, no. 5-6, pp. 320-340, 2012.

[4] J. Sosik, S. Kahai, and M. Piovoso, "Silver bullet or voodoo statistics? A primer for using the partial least squares data analytic technique in group and organization research," Group \& Organization Management, vol. 34, no. 1, pp. 5-36, 2009.

[5] B. Morard, C. Jeannette, and A. Stancu, "PLS Assistant," Université de Genève, Switzerland, 2007.
[6] Optimal-pls.com. (2016). Optimal PLS. [Online]. Available: http://optimal-pls.com/

[7] H. Wold, "Estimation of principal components and related models by iterative least squares," Multivariate Analysis, pp. 391-420, 1966.

[8] M. Tenenhaus, La régression PLS, 1st ed. Paris: Éd. Technip, 1998.

[9] M. Tenenhaus, V. Esposito Vinzi, Y. Chatelin, and C. Lauro, "PLS path modeling," Computational Statistics \& Data Analysis, vol. 48, no. 1, pp.159-205, 2005.

[10] S. Vancolen, "La régression PLS, " post grade diploma in statistics, University of Neuchâtel, Switzerland, 2004.

[11] J. Henseler, C. Ringle, and R. Sinkovics, "The use of partial least squares path modeling in international marketing," New Challenges to International Marketing Advances in International Marketing, vol. 20, pp. 277-319, 2009.

[12] V. Fernandes, "En quoi l'approche PLS est-elle une méthode a (re)découvrir pour les chercheurs en management?,"M@n@gement, vol. 15, no. 1, p. 102, 2012.

[13] B. Morard and A. Stancu, "Structural equation modeling in a rationalization tentative of balanced scorecard," Geneva: Cahier de Recherches de la Faculté de SES, HEC Genève, University of Geneva, Switzerland, 2005

[14] G. DeVault. (2007). Using Structural Equation Modeling (SEM) Cross-Sectional Statistical Modeling. The Balance. [Online] Available:

http://marketresearch.about.com/od/market.research.techniques/a/Usi ng-Structural-Equation-Modeling.htm

[15] P. Lei and Q. Wu, "Introduction to Structural Equation Modeling: Issues and Practical Considerations," Educational Measurement: Issues and Practice, vol. 26, no. 3, pp. 33-43, 2007.

[16] S. MacLean and K. Gray, "Structural equation modelling in market research," Journal of the Australian Market Research Society, vol. 6, pp. 17-32, 1998.

[17] G. Medsker, L. Williams, and P. Holahan, "A review of current practices for evaluating causal models in organizational behavior and human resources management research," Journal of Management, vol. 20, no. 2, pp.439-464, 1994.

[18] J. Hox and T. Bechger, "An introduction to structural equation modeling," Family Science Review, vol. 11, pp. 354-373, 1994.

[19] B. Morard and D. Simonin, "Partial least squares modeling in marketing research: A tailor-made model of wine e-commerce consumers in Switzerland," Journal of Economics, Business, and Management, vol. 4, no. 5, pp. 410-417, 2016.

[20] K. Wong, "Partial least squares structural equation modeling (PLSSEM) techniques using SmartPLS," Marketing Bulletin, vol. 24, pp. 1-32, 2013.

[21] H. Hwang, N. Malhotra, Y. Kim, M. Tomiuk, and S. Hong, "A comparative study on parameter recovery of three approaches to structural equation modeling," Journal of Marketing Research, vol. 47, no. 4, pp.699-712, 2010.

[22] V. E. Vinzi, W. Chin, J. Henseler, and H. Wang, Handbook of Partial Least Squares: Concepts, Methods and Applications, 1st ed. Berlin: Springer Handbooks of Computational Statistics, pp. 47-82, 2010.

[23] B. Morard, "Advanced controlling," Course of Master in Research, Faculty of Economics and Management, University of Geneva, Spring semester 2014.

[24] C. Werts, R. Linn, and K. Joreskog, "Intraclass reliability estimates: Testing structural assumptions," Educational and Psychological Measurement, vol. 34, no. 1, pp. 25-33, 1974.

[25] C. Fornell and D. Larcker, "Evaluating structural equation models with unobservable variables and measurement error," Journal of Marketing Research, vol. 18, no. 1, pp. 39-50, 1981.

[26] W. Chin, "The partial least squares approach to structural equation modeling," Modern Methods for Business Research, pp. 295-336, 1998.

[27] B. Morard, C. Jeannette, and A. Stancu, "The relationship between Structural Equation Modeling and Balanced Scorecard: Evidence from a Swiss non-profit organization," Review of Business \& Financial Studies, vol. 3, no. 2, pp. 21-36, 2012.

[28] P. Bastien, V. E. Vinzi, and M. Tenenhaus, "PLS generalised linear regression," Computational Statistics \& Data Analysis, vol. 48, no. 1, pp.17-46, 2005.

[29] B. Morard, A. Stancu, and C. Jeannette, "Finding your company's optimal balanced scorecard: A new quality criterion," Economics and Finance Research, vol. 61, pp. 65-74, 2013.

[30] R. Kaplan and D. Norton, "Using the balanced scorecard as a strategic management system," Harvard Business Review, JanuaryFebruary, pp. 75-85, 1996.

[31] F. Noyan and G. Simsek, "A partial least squares path model of repurchase intention of supermarket customers," Procedia - Social and Behavioral Sciences, vol. 62, pp. 921-926, 2012. 


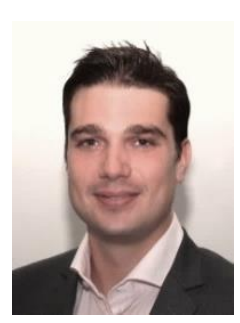

Dimitri Simonin received a master of science degree with a major in strategy from the Faculty of Economics and Management at the University of Geneva (Switzerland) in 2015, a WSET® Award Level 2 in wines and spirits (Fribourg/Switzerland) in 2015, a wealth management diploma from the UBS Business University (Zürich/Switzerland) in 2011.

He has worked as a portfolio advisor at UBS Wealth Management \& Swiss Bank (Geneva/Switzerland) and also operated as a strategic marketing consultant in the marketing department of a wine importing company (Geneva/Switzerland). At present, he is working as research partner with Prof. Morard, at the University of Geneva (Geneva/Switzerland), and he is pursuing a $\mathrm{PhD}$ focused on the wine business, including e-consumer behavior, importance of knowledge, the tourism business, and stakeholders' partnership efficiency, at the University of Technology Sydney (UTS), in Sydney (NSW, Australia).

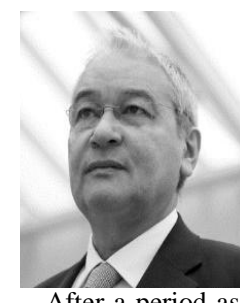

Bernard Morard is a double Ph.D. in finance and management accounting. He specialized in the financial and management accounting field, teaching at prestigious schools such as the University of Law, the Faculty of Applied Economics in Aix-enProvence and thereafter at the University of Montréal. He is the author of numerous publications in his field. He has been full professor at the University of Geneva, Switzerland since 1990

After a period as president of the HEC business school, he was elected the dean of the Faculty of Social and Economic Sciences at the University of Geneva in 2009. Using his entrepreneurial approach, he created more than 30 economic programs for professionals in various sectors during the last decade. His two $\mathrm{PhDs}$ made him very polyvalent in terms of research; his fields of research are focused on specific statistical tools for a better understanding of performance in macro and micro sectors. His favorite computational tools are Matlab, SPSS, Mathematica, and Free Basic. 